\title{
Transcriptome network analysis of potential candidate genes for heart failure
}

\author{
J. Chen ${ }^{1,2}$, H.Y. Wang ${ }^{1,2}$ and C.Y. Zeng ${ }^{1,2}$ \\ ${ }^{1}$ Department of Cardiology, Daping Hospital, \\ Third Military Medical University Cardiology, Chongqing, China \\ ${ }^{2}$ Chongqing Institute of Cardiology, Chongqing, China \\ Corresponding author: C.Y. Zeng \\ E-mail: chunyuzeng@hotmail.com
}

Genet. Mol. Res. 12 (4): 4687-4697 (2013)

Received November 24, 2012

Accepted March 15, 2013

Published October 18, 2013

DOI http://dx.doi.org/10.4238/2013.October.18.7

\begin{abstract}
The purpose of this study was to examine the hypothesis that a transcriptome network can be developed through a set of transcription factors regulated by the expression of various genes induced by dilated cardiomyopathy can be identified and modulated to respond to heart failure. We searched for significant pathways related to dilated cardiomyopathy using the GSE4172 microarray data to identify potential genes related to heart failure. We mapped differentially expressed genes to pathways and constructed a regulation network to investigate the regulatory relationships between transcription factors and pathways. Some transcription factors and target genes in the networks have been clearly linked to heart failure in previous studies. We also found new transcription factors and target genes, such as CCAAT/ enhancer-binding protein delta and JunB, responsible for inflammatory cardiomyopathy. Transcriptome network analysis was useful in the identification of candidate genes in heart failure. This method is well suited for microarray data and therefore is proposed as a powerful tool in the search for new pathways related to disease.
\end{abstract}

Key words: Transcriptome network; Candidate genes; Heart failure 


\section{INTRODUCTION}

Heart failure (HF) is generally defined as the inability of the heart to supply sufficient blood flow to meet bodily needs. In different contexts, HF is often called congestive HF or acute decompensated HF. Several diseases are closely related to and may cause HF - for example, dilated cardiomyopathy (DCM). Inflammatory cardiomyopathy (DCMi) is an important DCM subtype often found in conjunction with cardiac viral infections and characterized by dilation and dysfunction of the ventricles (Wittchen et al., 2007).

Recently the mechanism of HF has been summarized as follows. A variety of neurohormonal proteins, including norepinephrine, angiotensin II, endothelin, aldosterone, and tumor necrosis factor (TNF), have been implicated as some of the biologically active molecules that contribute to disease progression in the failing heart. Oxidant signaling is increased in HF-for example, that of superoxide, hydrogen peroxide, hydroxyl radical, and nicotinamide adenine dinucleotide phosphate-oxidase. Furthermore, TNF- $\alpha$ exerts strong direct effects on cardiomyocytes, inducing apoptosis, depressing contractility, and downregulating sarcomeric proteins. Cardioprotection mechanisms are mediated by interleukin (IL)-6, glycoprotein 130, and signal transducer and activator of transcription 3 signaling (Mann and Bristow, 2005; Hilfiker-Kleiner, et al., 2006).

DNA microarray analysis is a global approach to investigating physiological mechanisms in health and disease (Spies et al., 2002). A high-throughput microarray experiment has been designed to analyze genetic expression patterns and identify potential genes to target HF (Xu et al., 2011). Genomic expression profiling has also evolved as a useful tool for identifying novel pathomechanisms in human cardiac disorders (Verducci et al., 2006).

The purpose of this study was to examine the hypothesis that a transcriptome network can be developed in which a set of transcription factors (TFs) regulating the expression of various genes induced by DCM can be identified and modulated to respond to HF. Further analysis of these genes and pathways in the network was carried out to identify potential mechanisms that respond to HF. This study did not address the regulation network but searched for the significant pathways related to DCM.

\section{MATERIAL AND METHODS}

\section{Data}

\section{Affymetrix microarray data}

Transcription profiles of ischemic cardiomyopathy GSE4172 (Wittchen et al., 2007) were obtained from the public functional genomics data repository Gene Expression Omnibus (http:// www.ncbi.nlm.nih.gov/geo/). Only 12 chips were usable. Samples derived from 8 DCMi and 4 healthy control patients were hybridized onto Affymetrix U133 Plus arrays (Wachi et al., 2005).

\section{Pathway data}

The Kyoto Encyclopedia of Genes and Genomes (KEGG) is a collection of online databases of with genomes, enzymatic pathways, and biological chemicals (Kanehisa, 2002). The PATHWAY database records networks of molecular interactions in cells and variants spe- 
cific to particular organisms (http://www.genome.jp/kegg/). A total of 130 pathways involving 2287 genes were collected from KEGG.

\section{Regulation data}

Approximately 2600 proteins in the human genome contain DNA-binding domains, and most are presumed to function as TFs (Kanehisa, 2002; Wachi et al., 2005). The combinatorial use of a subset of the approximately 2000 human TFs easily accounts for the unique regulation of each gene in the human genome during development (Brivanlou and Darnell Jr., 2002).

These TFs are grouped into 5 superclass families based on the presence of conserved DNA-binding domains. The Transcription Factor (TRANSFAC; http://www.gene-regulation. $\mathrm{com} /$ pub/databases.html) database contains data on TFs, their experimentally proven binding sites, and regulated genes (Wingender, 2008).

The Transcriptional Regulatory Element Database (TRED; http://rulai.cshl.edu/ $\mathrm{TRED} /$ ) was built in response to increasing needs for an integrated repository for both cis and trans-regulatory elements in mammals (Jiang et al., 2007). TRED supplies curation for transcriptional regulation information, including TF binding motifs and experimental evidence. The curation is currently focusing on target genes of 36 cancer-related TF families.

A total of 774 pairs of regulatory relationships between 219 TFs and 265 target genes were collected from TRANSFAC and 5722 pairs of regulatory relationships between 102 TFs and 2920 target genes were collected from TRED. Combining the data from both data sets yielded 6328 regulatory relationships between 276 TFs and 3002 target genes (Table 1).

$\begin{aligned} & \text { Table 1. Regulation data form Transcription Factor (TRANSFAC) and Transcription Regulatory Element } \\
& \text { Database (TRED). }\end{aligned}$
\begin{tabular}{lcccl} 
Source & Regulation & TFs & Targets & Link \\
\hline TRANSFAC & 774 & 219 & 265 & $\mathrm{http} / /$ www.gene-regulation.com/pub/databases.html \\
TRED & 5722 & 102 & 2920 & $\mathrm{http} / /$ rulai.cshl.edu/TRED/ \\
Total & 6328 & 276 & 3002 & \\
\hline
\end{tabular}

TFs $=$ transcription factors.

\section{Methods}

\section{Differentially expressed gene (DEG) analysis}

Because the original CEL file did not exist for the GSE4172 data set, we analyzed only processed data. All statistical analyses were accomplished using $\mathrm{R}$ program. We compared the disease group with the control group. A $t$-test (Koch and Spörl, 2007) was used to identify DEGs. A P value of 0.05 was chosen as the cut-off significance level. DEGs with fold change values larger than 2 were selected.

\section{Co-expression analysis}

To demonstrate potential regulatory relationships, we calculated the Pearson correlation coefficient (PCC) for all pairwise comparisons of gene expression values between TFs and DEGs. The regulatory relationships with absolute PCCs larger than 0.6 were considered significant. 


\section{Gene Ontology (GO) analysis}

The Biological Networks Gene Ontology tool is an open-source Java tool to determine which GO terms are significantly overrepresented in a set of genes. The Biological Networks Gene Ontology analysis (Maere et al., 2005) was used to identify over-represented GO categories in biological processes.

\section{Regulation network construction}

Using the regulation data collected from the TRANSFAC and TRED databases, we matched the relationships between differentially expressed TFs and their target DEGs. Using the data from the 2 regulation data sets and the pathway relationships of the target genes, we constructed the regulation networks using Cytoscape (Shannon et al., 2003). Bases on the significant relationships ( $\mathrm{PCC}>0.6$ or PCC $<-0.6$ ) between TFs and target genes, 33 putative regulatory relationships were predicted between $7 \mathrm{TFs}$ and 22 target genes.

\section{Significance analysis of pathway}

We adopted an impact analysis that not only included the statistical significance of the set of pathway genes but also considered other crucial factors such as the magnitude of each gene expression change, the topology of the signaling pathway, genes and signaling pathways interactions, and others (Draghici et al., 2007). In this model, the impact factor (IF) of a pathway Pi was calculated as the sum of 2 terms:

$$
I F(P i)=\log \left(\frac{1}{p i}\right)+\frac{\sum_{g \in P_{i}}|P F(g)|}{|\Delta E| \cdot N_{\dot{x}}(P i)}
$$

The 1 st term is probabilistic and captures the significance of the given pathway, $P i$, from the perspective of the set of genes contained in it. It is obtained using the hyper geometric model in which $p i$ is the probability of obtaining at least the observed number of DEGs $\left(N_{d e}\right)$ by chance (Tavazoie et al., 1999; Draghici et al., 2003). The 2 nd term is functional and depends on the identity of the DEGs as well as the interactions described by the pathway (i.e.; its topology). This term sums up the absolute values of the perturbation factors (PFs) for all genes $g$ on the given pathway $P i$.

The PF of a gene $g$ is calculated as follows:

$$
P F(g)=\Delta E(g)+\sum_{u \in U S g} \beta_{u g} \cdot \frac{P F(u)}{N_{d s}(u)}
$$

Where the 1st-term $\Delta E(g)$ captures the quantitative information measured in the gene expression experiment. The factor $\Delta E(g)$ represents the normalized measured expression change of a gene $g$. The 1st-term $\Delta E(g)$ in the above equation is a sum of all PFs of genes $u$ directly upstream of the target gene $g$, normalized by the number of downstream genes of each such gene, $N_{d s}(u)$, and weighted by the factor $\beta_{u g}$, which reflects the type of interaction: 
$\beta_{u g}=1$ for induction, $\beta_{u g}=-1$ for repression (KEGG supplies this information about the type of interaction of 2 genes in descriptions of pathway topology). US $S_{g}$ is the set of all such genes upstream of $g$. We normalized the calculation with respect to the size of the pathway by dividing the total perturbation by the number of DEGs on the given pathway, $N_{d e}\left(P_{i}\right)$. To make the IFs as independent as possible from the technology and also comparable between investigations, we divided the 2 nd term in equation 1 by the mean absolute fold change, $\Delta E$, calculated across all DEGs. The result of the significance analysis of the pathways is shown in Table 2.

\begin{tabular}{|c|c|c|c|c|}
\hline Database name & Pathway name & Impact factor & $\%$ Pathway genes in input & $\mathrm{P}$ \\
\hline KEGG & ANKRD1 & 191.843 & 11.765 & 0.07426 \\
\hline KEGG & ANKRD1 & 58.047 & 8.209 & 0.447219 \\
\hline KEGG & Antigen processing and presentation & 42.358 & 5.618 & 0.773023 \\
\hline KEGG & Adherens junction & 37.643 & 10.256 & 0.267516 \\
\hline KEGG & Phosphatidylinositol signaling system & 32.239 & 14.474 & 0.040482 \\
\hline KEGG & Regulation of actin cytoskeleton & 10.907 & 15.207 & $1.21 \mathrm{E}-04$ \\
\hline KEGG & Thyroid cancer & 8.015 & 17.241 & 0.079416 \\
\hline KEGG & Tight junction & 6.779 & 12.593 & 0.034159 \\
\hline KEGG & Melanoma & 6.766 & 15.493 & 0.02602 \\
\hline KEGG & Gap junction & 6.353 & 11.458 & 0.127498 \\
\hline
\end{tabular}

\section{Regulation network between TFs and pathways}

To investigate further the regulatory relationships between TFs and pathways, we mapped DEGs to pathways and obtained a regulation network between TFs and pathways (Figure 1).

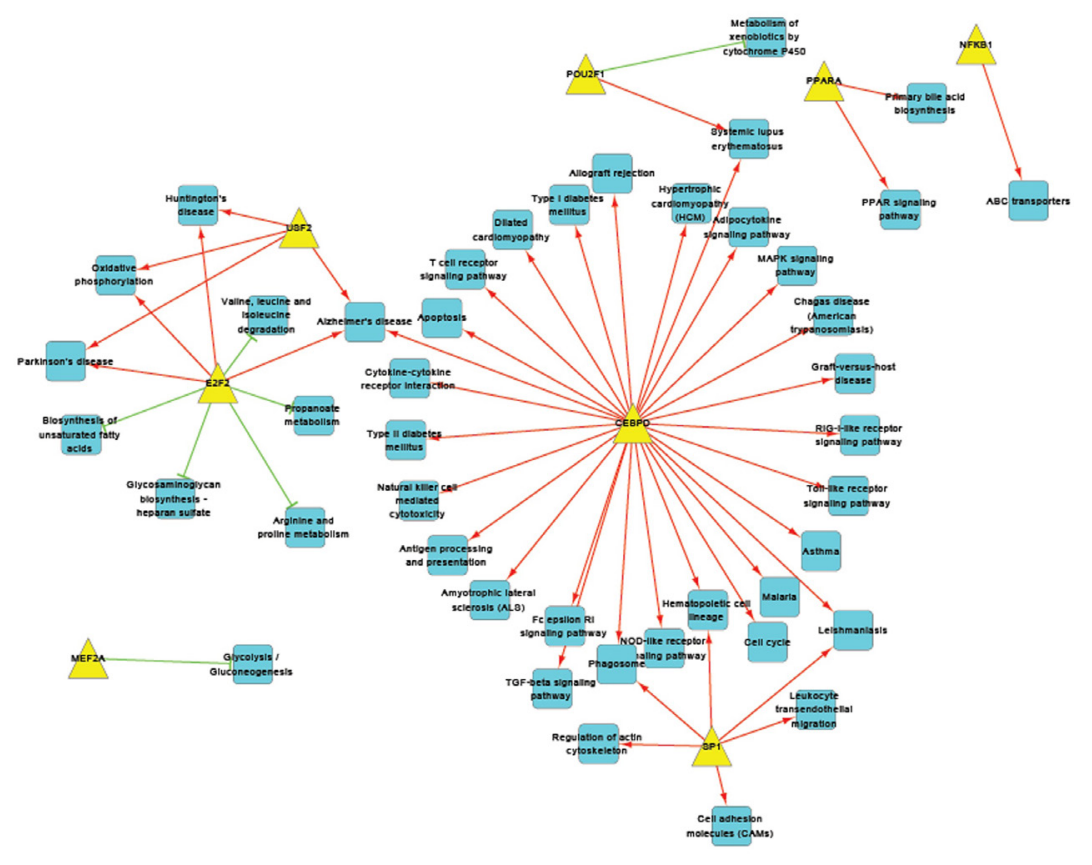

Figure 1. Regulation network construction in heart failure. 


\section{RESULTS}

\section{Regulation network construction in $\mathrm{HF}$}

To get pathway-related DEGs for HF, we obtained the publicly available microarray data set GSE4172 from the Gene Expression Omnibus. After microarray analysis, the DEGs with fold change values larger than 2 and $P$ values less than 0.05 were used. We selected 2156 genes as DEGs from GSE4172. To determine the regulatory relationships, we chose the co-expressed value PCC $\geq 0.6$ as the threshold. Finally, we obtained 37 regulatory relationships between 13 differentially expressed TFs and their 36 target DEGs. By integrating the regulatory relationships above, a regulation network of HF was built between TFs and their target genes (Figure 2). In this network, E2F transcription factor 2 (E2F2), CCAAT/enhancer binding protein (CEBPD), and POU class 2 homeobox 1(POU2F1) with higher PCC value formed a local network, which suggests that these TFs may play important roles in HF. Furthermore, the integrin alpha M (ITGAM) target gene regulated by specificity protein 1 was observed in this network.

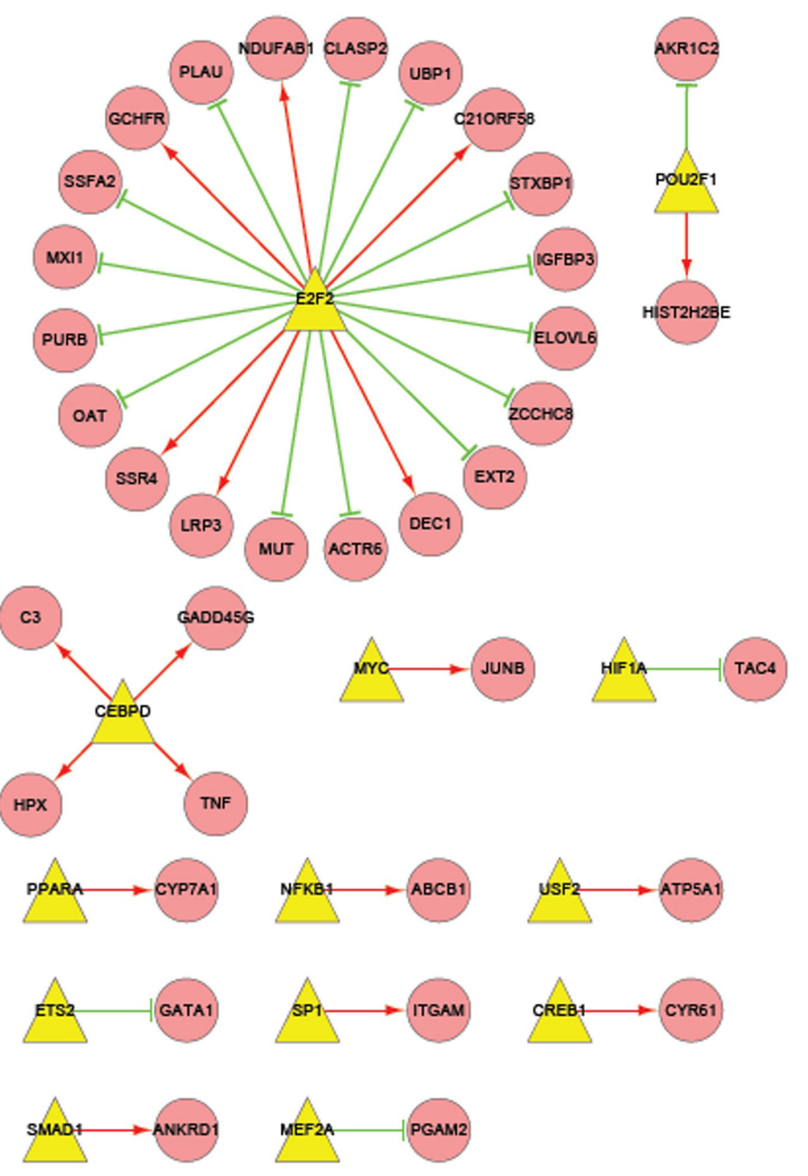

Figure 2. Regulation network between transcription factor and PATHWAY. 


\section{GO analysis of the regulation network in HF}

Several GO categories were enriched among these genes in the regulatory network, including regulation of transcription from RNA polymerase II promoter, positive regulation of transcription, regulation of transcription, DNA-dependent and regulation of RNA metabolic process. Table 3 lists the top 10 categories.

\begin{tabular}{|c|c|c|c|c|}
\hline GO-ID & Description & Count & $\mathrm{P}$ & Corr P \\
\hline 6357 & Regulation of transcription from RNA polymerase II promoter & 14 & 5.02E-08 & $6.72 \mathrm{E}-05$ \\
\hline 45941 & Positive regulation of transcription & 12 & $1.77 \mathrm{E}-07$ & 8.57E-05 \\
\hline 6355 & Regulation of transcription, DNA-dependent & 20 & $2.06 \mathrm{E}-07$ & 8.57E-05 \\
\hline 10628 & Positive regulation of gene expression & 12 & $2.96 \mathrm{E}-07$ & 8.57E-05 \\
\hline 51252 & Regulation of RNA metabolic process & 20 & $3.20 \mathrm{E}-07$ & $8.57 \mathrm{E}-05$ \\
\hline 45935 & $\begin{array}{l}\text { Positive regulation of nucleobase, nucleoside, nucleotide, and nucleic } \\
\text { acid metabolic process }\end{array}$ & 12 & 7.27E-07 & $1.51 \mathrm{E}-04$ \\
\hline 10604 & Positive regulation of macromolecule metabolic process & 14 & $8.53 \mathrm{E}-07$ & $1.51 \mathrm{E}-04$ \\
\hline 10557 & Positive regulation of macromolecule biosynthetic process & 12 & $9.83 \mathrm{E}-07$ & $1.51 \mathrm{E}-04$ \\
\hline 51173 & Positive regulation of nitrogen compound metabolic process & 12 & $1.01 \mathrm{E}-06$ & $1.51 \mathrm{E}-04$ \\
\hline 31328 & Positive regulation of cellular biosynthetic process & 12 & $1.94 \mathrm{E}-06$ & 2.53E-04 \\
\hline
\end{tabular}

\section{Significant pathways in HF}

To identify the relevant pathways changed in HF, we used a statistical approach on the pathway level. Significance analysis at the single-gene level can be disadvantaged by the limited number of samples and experimental noise, which severely reduce the power of the chosen statistical test. Pathways can provide an alternative way to relax the significance threshold applied to single genes and may lead to a better biological interpretation. Therefore, we adopted a pathway-based impact analysis method with many factors, including the statistical significance of the set of DEGs in the pathway, the magnitude of each gene expression change, the topology of the signaling pathway, their interactions, and others. The impact analysis method yielded many significant pathways containing leukocyte transendothelial migration, cell adhesion molecules (CAMs), antigen processing and presentation, and others (Table 2).

\section{Regulation network between TFs and pathways in HF}

To investigate further the regulatory relationships between TFs and pathways, we mapped DEGs to pathways and constructed a regulation network between the TFs and the pathways (Figure 1). In the network, CEBPD, E2F2, upstream transcription factor 2, spleen focus forming virus (SFFV) proviral integration oncogene spil (SPI), and POU2F1 were hub nodes linked to many HF pathways.

\section{DISCUSSION}

Much of the biological diversity of disease is the result of variation in transcriptional programs. Transcriptional profiling of disease using microarrays has revolutionized the field by allowing researchers to discover tumor subclasses and target genes for diagnosis and thera- 
py. Network and pathway analysis can be used interrogate gene expression data to understand the biological processes affected by a particular manipulation or disease/condition of interest. Transcriptome network analysis using a TF-target gene relationship has suggested, for example, that the genes upregulated in squamous lung carcinomas are rich in hubs. These hubs include E2F2, CEBPD, POU2F1, purine-rich element binding protein B (PURB), ITGAM, insulin-like growth factor binding protein 3 (IGFBP3), core-binding factor beta (CBFbeta), and ankyrin repeat domain-containing protein 1.

E2F2 protein is a member of the E2F family of TFs. It has an extra cyclin-binding domain and plays a crucial role in the control of the cell cycle. The expression of E2F2 has been mediated in the hearts of mice using adenoviral vectors, and E2F2 induces proliferation of cardiomyocytes, which may be used for HF treatment (Ebelt et al., 2008).

CEBPD protein is a bZIP TF that binds as a homodimer to certain DNA regulatory regions to regulate genes involved in immune and inflammatory responses. Atrial fibrillation is the most frequently encountered arrhythmia in the clinical setting. However, arrhythmia often leads to acute HF. CEBPD messenger RNA expression has been induced in the atria of AF patients (Ohki et al., 2005). Transgenic mice major histocompatibility complex (MHC)CEBPD $\triangle$ with inhibited CEBPD splicing activity in the heart leads to a severe form of cardiomyopathy characterized by specific defects in the splicing of CEBPD targets, cardiac hypertrophy, DCM, myocytolysis, fibrosis, changes in gene expression associated with HF, severe cardiac dysfunction, and premature death (Terenzi et al., 2009).

POU2F1, also known as organic cation transporter 1, is a TF that was among the first identified members of the POU TF family. Increased organic cation transporter 1 activity inhibits heart inflammation in heart shock treatment (Chen and Currie, 2005).

PURB is a single-stranded nucleic acid-binding protein. In humans, considerable loss of $\alpha$-myosin heavy chain $(\alpha-\mathrm{MHC})$ content has been implicated in reduced myocardial contractility during HF. PURB can participate in transcriptional as well as translational regulation of $\alpha$-MHC gene expression. Furthermore, robust expression of PURB has been expressed robustly in the myocardium of humans with HF. Therefore, PURB may play the role in the downregulation of $\alpha$-MHC expression in HF (Gupta et al., 2003).

The ITGAM gene encodes the integrin alpha $M$ chain. Alpha $M$ beta 2 integrin is important in the adherence of neutrophils and monocytes to stimulated endothelium and in the phagocytosis of complement-coated particles. Depletion of macrophages through treatment with anti-ITGAM monoclonal antibodies prevents encephalomyocarditis virus-induced myocarditis completely and inhibits viral growth in target organs (Hirasawa et al., 1996). When ITGAM monoclonal antibody is applied to heart interstitial dendritic cells, myocarditis subsides (Portella and Andrade, 2009).

IGFBP3 is a member of the IGFBP family and forms a ternary complex with insulinlike growth factor (IGF) acid-labile subunit and either IGF I or II. In this form, it circulates in the plasma, prolonging the half-life of IGFs and altering their interaction with cell surface receptors. IGFBP3 messenger RNA is abundantly expressed in patients with HF such as cardiomyopathy (Granata et al., 2000). The ratio of IGF-I to IGFBP3 is a predictor of clinical outcomes in HF. HF patients display significantly decreased serum IGF axis values (Watanabe et al., 2010).

In muscle cells, a complex network of Z-disc proteins allows proper reception, transduction, and transmission of mechanical and biochemical signals. Mutations in genes encoding vari- 
ous Z-disc proteins have recently been implicated in HF. CBFbeta is an essential component for maintaining sarcomeric Z-disc and myofilament organization in heart muscle. Transcription of the gene encoding CBFbeta mainly depends on JunB activity. JunB-morphant zebrafish show a heartfailure phenotype similar to that of CBFbeta-deficient zebrafish (Meder et al., 2010).

Ankyrin repeat domain-containing protein 1 functions as a TF localized to the nucleus of endothelial cells and induced by IL- 1 and TNF- $\alpha$ stimulation. This protein interacts with the sarcomeric proteins myopalladin and titin in the myofibrillar stretch-sensor system. It is upregulated in cardiac failure, causing contractile dysfunction that in turn causes DCM (Moulik et al., 2009).

The regulation network between TFs and pathways in HF showed that many pathways closely related to the disease were linked using our method. These pathways included CAMs and antigen processing and presentation pathways. In DCM hearts, the phenotypic pattern of endothelial cells is reportedly altered with respect to CAMs, such as selectins, immunoglobulin, and integrins. Selectins induce the rolling and sticking of leukocytes to endothelial cells. Adhesion molecules interact with specific integrins on various types of leukocytes and establish firm attachments to endothelial cells. These adhesion molecule-integrin interactions facilitate the migration of leukocytes through the endothelial layer into regions of inflammation.

In general, leukocyte transendothelial migration and CAM expression are associated with HF, which is a cascade process (Devaux et al., 1997; Noutsias et al., 1999; Golias et al., 2007). DCMi often results from cardiotropic viral infections such as parvovirus B19 and human herpesvirus 6. Autoimmune events are initiated under these conditions. A pathogen-induced breakdown in the control mechanisms protecting against autoimmune reactions through both the presentation of normal self-antigens and bystander activation leads to the formation of autoreactive antibodies and T cells. The auto-reactive antibodies interact directly with heart tissue, altering signal transduction or complement activation, whereas T-cell-mediated mechanisms include direct attack by cytotoxic $T$ cells or indirect effects of cytotoxic cytokines released by stimulated T cells or macrophages (Kallwellis-Opara et al., 2007).

A deeper understanding of TFs and their regulated genes remains an area of intense future research activity. Our regulation network will be useful in the investigation of complex interacting mechanisms of TFs and their regulated genes in disease. However, further experiments are still needed to confirm our conclusions.

Cardiomyopathy is one of the most frequent causes of HF and a leading indication for cardiac transplantation in the era of modern treatment. A search for new therapeutic targets in DCMi seems worthwhile owing to the high prevalence of this condition and the still unsatisfactory long-term outcome. The identification of HF-associated genes and their related pathways is essential to the opening of new avenues in the prevention of this disease. In this research, a transcriptome network was used to identify HF-associated genes and pathways. We also used factor analysis to determine whether a pathway is significantly changed in a microarray experiment. This method is well suited for microarray data and therefore is proposed as a powerful tool in the search for new pathways related to other diseases.

\section{REFERENCES}

Brivanlou AH and Darnell Jr JE (2002). Signal transduction and the control of gene expression. Science 295: 813-818. Chen Y and Currie RW (2005). Heat shock treatment suppresses angiotensin II-induced SP-1 and AP-1 and stimulates Oct-1 DNA-binding activity in heart. Inflamm. Res. 54: 338-343. 
Devaux B, Scholz D, Hirche A, Klovekorn WP, et al. (1997). Upregulation of cell adhesion molecules and the presence of low grade inflammation in human chronic heart failure. Eur. Heart J. 18: 470-479.

Draghici S, Khatri P, Martins RP, Ostermeier GC, et al. (2003). Global functional profiling of gene expression. Genomics 81: 98-104.

Draghici S, Khatri P, Tarca AL, Amin K, et al. (2007). A systems biology approach for pathway level analysis. Genome Res. 17: 1537-1545.

Ebelt H, Zhang Y, Kampke A, Xu J, et al. (2008). E2F2 expression induces proliferation of terminally differentiated cardiomyocytes in vivo. Cardiovasc. Res. 80: 219-226.

Golias C, Tsoutsi E, Matziridis A, Makridis P, et al. (2007). Review. Leukocyte and endothelial cell adhesion molecules in inflammation focusing on inflammatory heart disease. In Vivo 21: 757-769.

Granata R, Broglio F, Migliorino D, Cutrupi S, et al. (2000). Neonatal and adult human heart tissues from normal subjects and patients with ischemic, dilated or hypertrophic cardiomyopathy express insulin-like growth factor binding protein-3 (IGFBP-3). J. Endocrinol. Invest. 23: 724-726.

Gupta M, Sueblinvong V, Raman J, Jeevanandam V, et al. (2003). Single-stranded DNA-binding proteins PURalpha and PURbeta bind to a purine-rich negative regulatory element of the alpha-myosin heavy chain gene and control transcriptional and translational regulation of the gene expression. Implications in the repression of alpha-myosin heavy chain during heart failure. J. Biol. Chem. 278: 44935-44948.

Hilfiker-Kleiner D, Landmesser U and Drexler H (2006). Molecular mechanisms in heart failure: focus on cardiac hypertrophy, inflammation, angiogenesis, and apoptosis. J. Am. Coll. Cardiol. 48: A56-A66.

Hirasawa K, Tsutsui S, Takeda M, Mizutani M, et al. (1996). Depletion of Mac1-positive macrophages protects DBA/2 mice from encephalomyocarditis virus-induced myocarditis and diabetes. J. Gen. Virol. 77 (Pt 4): 737-741.

Jiang C, Xuan Z, Zhao F and Zhang MQ (2007). TRED: a transcriptional regulatory element database, new entries and other development. Nucleic Acids Res. 35: D137-D140.

Kallwellis-Opara A, Dorner A, Poller WC, Noutsias M, et al. (2007). Autoimmunological features in inflammatory cardiomyopathy. Clin. Res. Cardiol. 96: 469-480.

Kanehisa M (2002). The KEGG database. Novartis Found. Symp. 247: 91-101.

Koch R and Spörl E (2007). Statistical methods for comparison of two measuring procedures and for calibration: analysis of concordance, correlation and regression in the case of measuring intraocular pressure. Klin. Monbl. Augenheilkd. 224: $52-57$.

Maere S, Heymans K and Kuiper M (2005). BiNGO: a Cytoscape plugin to assess overrepresentation of gene ontology categories in biological networks. Bioinformatics 21: 3448-3449.

Mann DL and Bristow MR (2005). Mechanisms and models in heart failure: the biomechanical model and beyond. Circulation 111: 2837-2849.

Meder B, Just S, Vogel B, Rudloff J, et al. (2010). JunB-CBFbeta signaling is essential to maintain sarcomeric Z-disc structure and when defective leads to heart failure. J. Cell Sci. 123: 2613-2620.

Moulik M, Vatta M, Witt SH, Arola AM, et al. (2009). ANKRD1, the gene encoding cardiac ankyrin repeat protein, is a novel dilated cardiomyopathy gene. J. Am. Coll. Cardiol. 54: 325-333.

Noutsias M, Seeberg B, Schultheiss HP and Kuhl U (1999). Expression of cell adhesion molecules in dilated cardiomyopathy: evidence for endothelial activation in inflammatory cardiomyopathy. Circulation 99: 2124-2131.

Ohki R, Yamamoto K, Ueno S, Mano H, et al. (2005). Gene expression profiling of human atrial myocardium with atrial fibrillation by DNA microarray analysis. Int. J. Cardiol. 102: 233-238.

Portella RS and Andrade SG (2009). Trypanosoma cruzi: parasite antigens sequestered in heart interstitial dendritic cells are related to persisting myocarditis in benznidazole-treated mice. Mem. Inst. Oswaldo Cruz 104: 1023-1030.

Shannon P, Markiel A, Ozier O, Baliga NS, et al. (2003). Cytoscape: a software environment for integrated models of biomolecular interaction networks. Genome Res. 13: 2498-2504.

Spies M, Dasu MR, Svrakic N, Nesic O, et al. (2002). Gene expression analysis in burn wounds of rats. Am. J. Physiol. Regul. Integr. Comp. Physiol. 283: R918-R930.

Tavazoie S, Hughes JD, Campbell MJ, Cho RJ, et al. (1999). Systematic determination of genetic network architecture. Nat. Genet. 22: 281-285.

Terenzi F, Brimacombe KR, Penn MS and Ladd AN (2009). CELF-mediated alternative splicing is required for cardiac function during early, but not later, postnatal life. J. Mol. Cell. Cardiol. 46: 395-404.

Verducci JS, Melfi VF, Lin S, Wang Z, et al. (2006). Microarray analysis of gene expression: considerations in data mining and statistical treatment. Physiol. Genomics 25: 355-363.

Wachi S, Yoneda K and Wu R (2005). Interactome-transcriptome analysis reveals the high centrality of genes differentially expressed in lung cancer tissues. Bioinformatics 21: 4205-4208.

Watanabe S, Tamura T, Ono K, Horiuchi H, et al. (2010). Insulin-like growth factor axis (insulin-like growth factor-I/ 
insulin-like growth factor-binding protein-3) as a prognostic predictor of heart failure: association with adiponectin. Eur. J. Heart Fail. 12: 1214-1222.

Wingender E (2008). The TRANSFAC project as an example of framework technology that supports the analysis of genomic regulation. Brief. Bioinform. 9: 326-332.

Wittchen F, Suckau L, Witt H, Skurk C, et al. (2007). Genomic expression profiling of human inflammatory cardiomyopathy (DCMi) suggests novel therapeutic targets. J. Mol. Med. 85: 257-271.

Xu J, Nie HG, Zhang XD, Tian Y, et al. (2011). Down-regulated energy metabolism genes associated with mitochondria oxidative phosphorylation and fatty acid metabolism in viral cardiomyopathy mouse heart. Mol. Biol. Rep. 38: 40074013. 